\title{
Essais de transmission cyclique de trypanosomes du groupe congolense
}

\author{
par L. MAILLOT
}

\begin{abstract}
RESUME
Des essais de transmission cyclique de Trypanosoma congolense ont été pratiqués avec différentes espèces de glossines et plusieurs espèces animales. Les résultats positifs obtenus, bien que peu nombreux, fournissent cependant des indications utiles pour les recherches à venir
\end{abstract}

Une souche de trypanosomes du groupe congolense est entretenue sur cobaye et souris dans le Service d'Entomologie et de Protozoologie Tropicales de l'I.E.M.V.T. depuis décembre 1967 à partir d'un stabilat (E.A.T.R.O. 325) ayant pour origine un broyat de $G$. pallidipes capturées dans la région nord du lac Victoria.

A partir de cette souche lauteur a fait des essais de transmission cyclique en utilisant comme animaux donneurs et récepteurs : cobaye, souris et lapin; comme vecteur trois espèces de tsé-tsé en élevage au laboratoire: $G$. morsitans, $G$. austeni et $G$. tachinoides.

\section{EXPERIMENTATION}

Les tsé-tsé : après leur repas sur un animal reconnu contaminé étaient nourries les jours suivants, soit d'emblée sur un animal neuf, soit temporairement sur le lapin ou la poule et ensuite sur l'animal à infecter. Plus de 1.600 mouches ont été utilisées dont environ un millier de $G$. morsitans, $261 G$. austeni et $265 G$. tachinoides, les trois quarts étaient des mâles et, sauf une centaine de G. morsitans, des mouches jeunes âgées d'environ 24 heures et à jeun depuis leur éclosion.

Les animaux: les neuf essais possibles de transmission (cobaye-cobaye, cobaye-lapin, cobaye-souris, lapin-cobaye, lapin-lapin, lapinsouris, souris-cobaye, souris-lapin, souris-souris) ont été réalisés de façon inégale. Cinq cents mouches ont été employées pour la transmission de cobaye à cobaye, les transmissions lapinsouris et souris-cobaye ont nécessité chacune moins de 100 mouches, toutes les autres transmissions chacune de 100 à 200 mouches.

\section{EXAMENS}

La recherche des trypanosomes a été faite après le dernier repas des tsé-tsé sur l'animal à infecter :

- chez la mouche, soit inmédiatement après ce repas, soit de préférence un ou deux jours plus tard;

- chez l'animal récepteur après une période suivant la piqûre de la mouche égale au minimum à la durée de l'incubation estimée, d'après les résultats obtenus, environ à 2 ou 3 semaines pour la souris, 1 à 3 semaines chez le cobaye et 3 à 4 semaines chez le lapin.

\section{RESULTATS}

Les résultats de ces examens ont permis d'estimer (dans les conditions des expériences réalisées), dans quelle mesure les mouches pouvaient s'infecter sur un animal contaminé, comment s'infectait l'animal exposé à des piqûres infectantes et quelles étaient les carac- 
téristiques principales des passages cycliques obtenus.

\section{Infection des mouches}

Le taux d'infection salivaire obtenu (c'est-àdire le pourcentage des mouches présentant des trypanosomes dans l'hypopharynx) est en général faible 3,4 p. 100 et variable suivant les tsé-tsé, leur âge, peut-être leur sexe et les animaux utilisés. Grosso modo il a été de plus de 4 p. 100 pour $G$. austeni, de 3 à 4 p. 100 pour $G$. morsitans, de moins de 1 p. 100 pour $G$. tachinoides. Une très grande abondance de trypanosomes dans le sang de l'animal donneur ne semble pas entraîner obligatoirement un taux d'infection salivaire plus élevé chez la mouche. Par ailleurs, il n'est pas exclu que la souche utilisée soit d'une transmissibilité médiocre ou faible à en juger les résultats obtenus par certains expérimentateurs (DAME et Mac KENZIE, HARLEY et WILSON), voir plus loin note 4 .

Dans l'ensemble chez l'espèce $G$. morsitans l'infection salivaire paraît un peu plus forte chez les mâles mais cette différence est minime. Chez des G. morsitans âgées (une centaine) il n'a pas été constaté d'infection salivaire mais un seul cas d'infection intestinale ancienne chez une femelle. Le taux d'infection salivaire est pratiquement le même chez les femelles vierges ou fécondées après le repas infectant. Chez les deux autres espèces $G$. austeni et $G$. tachinoides toute comparaison serait difficilement valable étant donné le petit nombre de femelles employées.

Les infections intestinales seules sans infection de la trompe sont en général plus nombreuses, sauf pour l'espèce $G$. morsitans et pour les femelles.

Le cycle d'évolution chez la mouche est au minimum d'après ces observations de 16 jours. La durée moyenne du cycle d'évolution complet chez la mouche n'a pu être exactement estimée, il paraît raisonnable de l'évaluer entre 20 et 25 jours; on constate assez souvent, entre un et deux mois après le repas infectant, une dégénérescence des trypanosomes situés dans l'intestin ou dans la trompe, caractérisée par un gonflement avec aspect de têtards et avec ou non perte de la motilité, mais cette dégénérescence peut survenir encore plus tôt. Elle a été observée le $27^{\circ}$ jour après la piqûre infec- tante chez une mouche qui 5 jours auparavant avait contaminé le lapin. Cette observation ne constitue pas une règle générale: chez une mouche examinée 64 jours après la piqûre infectante les trypanosomes étaient très abondants, sans aucun aspect de dégénérescence, dans I'intestin, l'hypopharynx et sur le labre; par ailleurs dans les passages cycliques réalisés la mouche s'est montrée infectieuse de 21 à -18 jours après la piqûre de l'animal donneur.

L'infection des mouches paraît également liée à d'autres facteurs tels que les préférences trophiques de l'insecte et sa longévité (dans les conditions de l'expérimentation).

\section{Préférences trophiques}

$G$. morsitans s'est nourrie sur tous les animaux, et sur la souris en particulier toutes se gorgent abondamment et très vite.

G. austeni se nourrit irrégulièrement sur les animaux d'expérience.

$G$. tachinoides se nourrit sans difficulté sur le lapin, irrégulièrement sur la souris et sur le cobaye.

\section{Longévité}

Une mortalité trop élevée après le repas infectant peut avoir une influence défavorisante sur laptitude de la mouche à s'infecter, ce qui semble ici le cas de $G$. tachinoides chez qui la mortalité semble dépendre des premiers repas de la mouche. Cette espèce nourrie en premier lieu sur le lapin n'a pas un taux de mortalité supérieur à celui de $G$. morsitans et de $G$. austeni. Les premiers repas pris sur souris ou sur cobaye entraînent un abaissement notable de la longévité et il est assez difficile d'estimer dans ces conditions le degré réel d'infectibilité de cette espèce.

\section{Transmissions cycliques réalisées}

(Voir schéma et notes.)

Le taux d'infection salivaire est différent suivant l'animal donneur: il varie de 4,4 p. 100 pour les mouches infectées sur le cobaye, à 1,5 p. 100 pour celles infectées sur le lapin et à 2,4 p. 100 pour celles infectées sur la souris.

La réussite du passage cyclique dépend peutêtre du vecteur mais surtout (dans les conditions des expériences réalisées) de la combinaison animal donneur et animal récepteur. Dans certains essais, tous ou presque tous les animaux 
placés au contact de mouches présentant une infection salivaire (comme l'ont révélé les dissections et examens des mouches) s'infectent, et dans d'autres essais les animaux piqués par des mouches infectées, ou s'infectent mal ou ne s infectent pas du tout. Les transmissions apparemment les plus faciles ont été lapin souris 2 fois, lapin lapin 1 fois, souris lapin 2 fois et cobaye lapin 2 fois. Les résultats ont été médiocres pour les transmissions cobaye-souris 3 fois et cobaye cobaye 1 fois; les transmissions ont échoué de souris à cobaye, lapin à cobaye et souris à souris.

Le cobaye infecte la mouche d'une façon constante mais à des taux faibles, il est par contre mauvais récepteur.

Le lapin infecte médiocrement la mouche mais les trypanosomes semblent très transmissibles sauf pour le cobaye, le lapin se comporte comme le meilleur récepteur des 3 espèces animales employées.

La souris infecte très peu de mouches, mais toutes ne sont pas gorgées ou le sont médiocrement, et la seule transmission réussie a été la transmission souris lapin.

L'infection transmise par voie cyclique a paru en général assez voisine de l'infection transmise par voie directe, mais l'incubation est toujours plus longue.

Le cobaye s'infecte le plus souvent par voie directe, (les échecs d'infection sont rares) il fait une affection semi-aiguë qui se termine par la mort, ou très rarement par la guérison.

Le lapin inoculé par voie cyclique s'infecte presque toujours, peut-être aussi par voie directe, son infection cst médiocre mais très transmissible au début, l'état général n’est pas modifié et l'animal guérit spontanément.

La souris s'infecte difficilement par voie cyclique, par voie directe les cas de résistance à 1'infection sont rarissimes, et l'incubation comme la survie sont brèves.

\section{CONCLUSIONS}

L'interprétation de toutes ces observations doit finalement permettre par un choix du vecteur, de l'animal donneur et récepteur d'envisager dans les expériences à venir de meilleures possibilités dans la réalisation de l'objet principal de ces recherches : la transmission cyclique continue de $T$. congolense.

\section{SUMMARY}

\section{Cyclical transmission of trypanosoma of the " congolense " group}

Some tests for the purpose of obtaining the cyclical transmission of Trypanosoma congolense have been carried out with different species of tsetse flies and some animal species. Though few in number, the positive results obtained will give some useful clues for the future research work.

\section{RESUMEN}

Ensayos de transmisión ciclica de tripanosomos del grupo «congolense "

Se efectuaron ensayos de transmisión ciclica de Trypanosoma congolense con diferentes especies de glosinas y varias especies animales. Los resultados obtenidos aunque sean poco numerosos sin embargo dan indicaciones útiles para las investigaciones venideras.

\section{BIBLIOGRAPHIE}

DAME (D. A.), MACKENZIE (P.K.I.), «Transmission de Trypanosoma congolense par des mâles de Glossina morsitans traités aux chimio-stérilisants » (Transmissions of Trypanosoma congolense by chemosterilized male Glossina morsitans). Ann. Trop. Med. Parasit., 1968, 62 (3): 372-374.

HARLEY (J. M.B.), WILSON (A. J.), « Comparaison entre Glossina morsitans, $G$, pallidipes et $G$. fuscipes comme vecteurs de trypanosomes du groupe de Trypanosoma congolense : les proportions d'infections expérimentales et les nombres d'organismes infectieux éliminés au cours du repas » (Comparison between Glossina morsitans, $G$. pallidipes and $G$. fuscipes as vectors of trypanosomes of the Trypanosoma congolense group: proportions infected experimentally and the numbers of infective organisms extruded during feeding). Ann Trop. Med. Parasit., 1968, 62 (2): 178-187. 


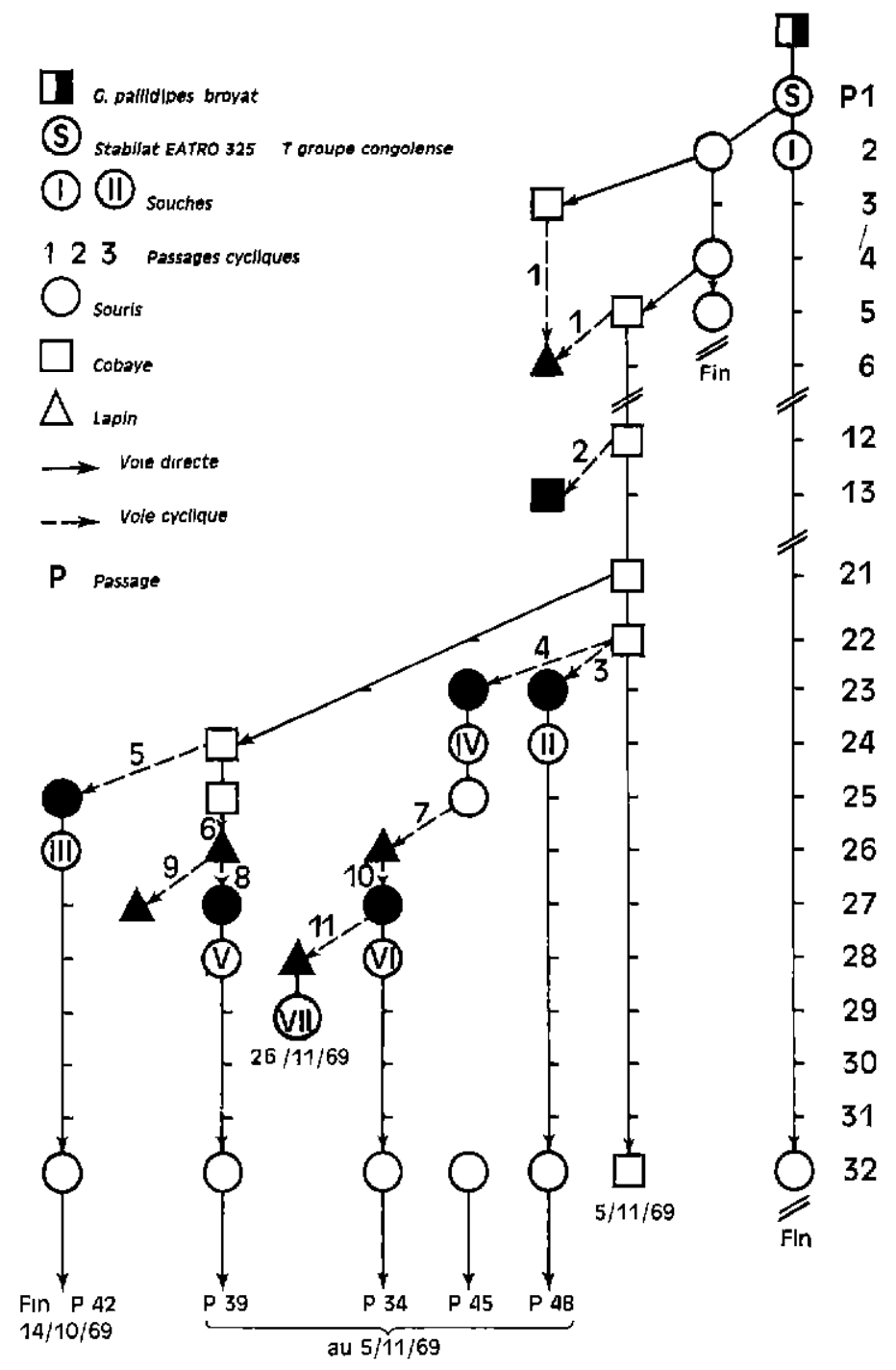




\section{PASSAGES CYCLIQUES REALISES (voir schéma)}

Passage $n^{\prime \prime} 1$ : mars 1968, de cobaye à lapin par des mâles et des femelles vierges de G. morsilans.

Passage n" 2 : septembre 1968, de cobaye à cobaye par des mâles de $G$. morsitans,

Passage no 3 : mars 1969, de cobaye à souris par des mâles de $G$. morsitans.

Passage no 4 : avril 1969 , du même cobaye à souris probablement par des mâles de $G$. tachinoides.

Passage $\mathbf{n}^{\circ} 5$ : avrıl 1969, de cobaye à lapin par des mâles de $G$. austeni.

Passage n" 6 : mai 1969, de cobaye à lapin par des mâles et des femelles fécondées de G. austeni.

Passage no 7 : juin 1969, de souris à lapin par des mâles de $G$. austeni.

Passage n" 8 : juin 1969 (succédant au passage no 6) de lapin à souris par des femelles fécondées de $G$. morsitans.

Passage n 9 : juillet 1969 (succédant au passage $\mathrm{n}^{\circ}$ 6) de lapin à lapin par les mêmes mouches qu'au passage $n^{0} 8$ : femelles fécondées de $G$. morsitans.

Passage no 10 : septembre 1969 (succédant au passage $\mathrm{n}^{0}$ 7) de lapin à souris par des mâles de $G$. morsitans.

Passage no 11: octobre 1969 (succédant au passage $n^{\circ} 10$ ) de souris à lapin par des mâles de $G$. morsitans.

\section{SOUCHES ISSUES DU STABILAT E A T.R.O. 325 ET ENTRETENUES AU LABORATOIRE PAR VOIE DIRECTE}

Actuellement il existe plusieurs souches en provenance de la souche originelle (vo1r schéma):

une souche cobaye-cobaye et 5 souches souris-souris, ces dernières sont toutes dans la filiation d'un ou plusieurs passages cycliques:

- dans la souche VII le trypanosome est passé 4 fois par la mouche, passages cycliques $n^{0 \leq 5} 4,7,10$ et 11 . dont 3 passages successifs 7,10 et 11 .

- dans la souche VI 3 fois, passages nov 4,7 et 10 dont 2 passages successifs $n^{\text {ws }} 7$ et 10 .

- dans la souche $V 2$ fois, passages $n^{13 \pi} 6$ et 8 successifs.

- dans la souche IV 1 fois, passage no 4.

- dans la souche II 1 fois, passage $\pi^{\circ} 3$.

Les deux souches VII et Vl passées respectivement quatre et trois fois par l'insecte, ne présentent pas une virulence accrue mais plutôt irrégulière.
La transmission a été arrêtée dans deux autres souches volontairement au 32c passage de la souche II entretenue par voie directe depuss le début et accidentellement au $42^{\circ}$ passage dans Ja souche III où le trypanosome était passé 1 fois par la souche (passage cyclique $n^{\prime \prime} 5$ ).

\section{CHIFFRES}

Mouches infectées suivant lanimal donneur et la mouche, sont présentés successivement le nombre des infections intestinales seules, le nombre des infections salivaires, le nombre des examens et le nombre des mouches non examinées ou examinées dans les 15 jours suivant la piqûre de l'animal infecté.

Cobaye $(50)+32 / 731+199=8(48)+24 / 607$ $+171 q(2)+8 / 124+28$

Lapin (11) $+4 / 272+53=\delta(7)+2 / 102$ $+23+$ 우 $+2 / 170+30$

Souris (17) $+6 / 245+122=\delta(14)+6 / 229$ $+92+$ o (3) $+0 / 23+30$

G. morsitans

$(27)+31 / 905+190=\delta(24)+25 / 645+137$ $+q(3)+6 / 260+53$

G. austeni

(32) $+10 / 208+53=8(29)+6 / 176+47$
$+9(3)+4 / 32+6$

\section{G. tachinoides}

(19) $+1 / 135+131=\delta(16)+1 / 110+102$ $+q(3)+0 / 25+29$

Total $(78)+42 / 1248+374=\delta(69)+32 / 931$ $+286+9(9)+10 / 317+88$

Coefficient d'infection salivaire p. 100 :

cobaye 4,4 ; lapin 1,5 ; souris 2,4

$G$. morsitans 3,$4 ; G$. atsteni 4,8 et $G$. tachinoides 0,74 .

Ces résultats sont à confronter à ceux d'expérimentations voisines publiées en 1968 par:

- HARLEY et WILSON qui signalent un pourcentage de mouches infectées dans la trompe et l'intestin de 11,6 p. 100 chez $G$. morsitans, de 13.2 p. 100 chez $G$. pallidlpes, de 2,9 p. 100 chez $G$. fuscipes, espèces nourries sur des animaux infectés par des trypanosomes du groupe congolense (vache, rat blanc, chèvre).

- par DAME et MacKENZIE qui, dans les lots de mouches témoins, $G$. morsitans, utilisées pour la transmission de cobaye à cobaye de $T$. congolense, trouvent un taux d'infection dans l'hypopharynx de 4,1 p. 100 , dans l'intestin de 31.5 p. 100 avec six transnissions réussies pour six pratiquées. 